\title{
Ictiofauna da praia de Jaguaribe, Itamaracá, Pernambuco
}

\author{
Ana Karla F. Lira \& Simone F. Teixeira
}

Laboratório de Ecologia de Peixes Tropicais, Universidade de Pernambuco, Rua Arnóbio Marques, 310, Santo Amaro, $50100-130$ Recife, PE. (redol@terra.com.br)

\begin{abstract}
Ichthyofauna of Jaguaribe beach, Itamaracá, Pernambuco, Brazil. Aiming to analyze the diversity of fish fauna on the beach of Jaguaribe, at Itamaracá island, four collections had been carried through April and November 2003, and March and August 2004, made with beach seine net. A total of 25 species and 24 genus were recorded comprised in seven orders and 15 families. The Perciformes order was most frequent representing $82.25 \%$ of the total of sampled fishes. It had predominance of representatives of the Sciaenidae (49.40\%), and the species most abundant was Ophioscion punctatissimus Meek \& Hildebrand, 1925 enclosing about $40.77 \%$ of the species total. For the analysis of diversity of Shannon, the month of November was what it got greater diversity (0.805) and equitability (0.843) and, the month of March, what it got the lesser diversity (0.562) and equitability (0.540). The density of fishes increases during dry periods suggesting that pluviometric variations could be structuring fish community at local scale.
\end{abstract}

KEYWORDS. Fish fauna, diversity, Jaguaribe Beach, northeastern Brazil.

RESUMO. Com o objetivo de analisar a diversidade da ictiofauna na praia de Jaguaribe, localizada na ilha de Itamaracá - PE, foram realizadas quatro coletas (abril e novembro de 2003 e março e agosto de 2004) com 10 arrastos cada, feitos com redes de arrasto. Um total de 25 espécies e 24 gêneros foram registrados compreendendo 7 ordens e 15 famílias. A ordem Perciformes foi a mais freqüente, representando $82,25 \%$ do total de peixes amostrados. Houve predominância dos representantes da família Sciaenidae $(49,40 \%)$ e a espécie mais abundante foi Ophioscion punctatissimus Meek \& Hildebrand, 1925, com aproximadamente 40,77\% do total das espécies. $\mathrm{Na}$ análise da diversidade de Shannon, o mês de novembro foi o que obteve maior diversidade $(0,805)$ e eqüitabilidade $(0,843)$ e o mês de março, o que obteve a menor diversidade $(0,562)$ e eqüitabilidade $(0,540)$. Uma tendência de maior abundância da ictiofauna foi observada no período chuvoso, sugerindo que as variações pluviométricas afetam a comunidade de peixes local.

PALAVRAS CHAVE. Ictiofauna, diversidade, praia de Jaguaribe, nordeste do Brasil.

A região costeira, embora muito menos extensa do que a região oceânica, apresenta uma produtividade muito maior. Essa maior produtividade se deve a uma maior absorção da energia solar, necessária à fotossíntese, visto que a extensão e a profundidade são menores na região costeira e a fotossíntese é responsável pela produtividade primária (PEREIRA \& SOARES-GoMEs, 2002).

Na costa da região Nordeste, características como a alta temperatura, alta salinidade e baixa concentração de nutrientes levam a uma produtividade primária muito baixa, com exceção dos estuários (Pereira \& SoaresGomes, 2002).

De acordo com Pereira \& SoARes-Gomes (2002), os peixes, importantes representantes da cadeia trófica sustentada pela produção primária, constituem a maior fração do nécton marinho, e a pesca marítima atualmente é responsável por $16 \%$ da proteína animal no consumo direto do homem, além de contribuir para a produção de ração animal utilizada na pecuária e na produção de tinta e fármacos.

A ictiofauna do canal de Santa Cruz vem sendo estudada sob vários aspectos, incluindo taxonomia, ecologia e piscicultura (ESKINAZI-LEÇA \& VASCONCELOS Filho, 1972; Cavalcanti, 1979; Vasconcelos Filho, 1979; Azevedo \& Guedes, 1980; Couto \& Vasconcelos Filho, 1980; Guedes \& Vasconcelos Filho, 1980; Cavalcanti $e t$ al., 1998; Vasconcelos Filho \& Oliveira, 1999; VAsconcelos Filho et al., 2003). A região costeira de
Pernambuco, contudo, ainda carece de estudos sobre a composição da ictiofauna (VASCONCELOS Filho et al., 2004).

Considerando a escassez de informações sobre a ictiofauna marinha costeira, o presente trabalho objetivou realizar o levantamento da ictiofauna da praia de Jaguaribe, litoral de Pernambuco, e analisar a sua diversidade nos períodos seco e chuvoso.

\section{MATERIAL E MÉTODOS}

A ilha de Itamaracá, localizada $50 \mathrm{~km}$ ao norte de Recife, fica separada do continente pelo canal de Santa Cruz e pelos rios que nele deságuam. Pela beleza, valor histórico e proximidade da capital do Estado, a área apresenta grande potencial turístico. O complexo estuarino de Itamaracá vem sendo alvo de ocupação indiscriminada por parte do setor industrial e turístico, além do aumento da densidade populacional (MACÊDO et al., 2000).

Por estar localizada na zona litoral e zona da mata, a área apresenta um clima tropical do tipo Am' com transição para As' no sistema de Köeppen e uma precipitação com balanço anual positivo. A temperatura do ar mostra máxima absoluta de $34^{\circ} \mathrm{C}$, mínima de $20^{\circ} \mathrm{C}$, com uma compensação média de $27^{\circ} \mathrm{C}$ (ANDRADE \& Lins, 1971). O regime sazonal apresenta uma repartição marcadamente tropical, com estação chuvosa de março a agosto e seca de setembro a fevereiro (MACÊDO et al., 2004). 
Dentre as praias que existem na ilha de Itamaracá está a de Jaguaribe, praia urbana caracterizada pela presença constante de grande quantidade de algas arribadas, localizada na região Nordeste da ilha.

Os peixes foram capturados com redes de arrasto de $5 \mathrm{~m}$ x 1,5 m e $8 \mathrm{~mm}$ entre nós em quatro coletas realizadas na praia de Jaguaribe ( $7^{\circ} 43,722^{\prime}$ S; 3449,536'O) (Fig. 1), em abril e novembro de 2003 e março e agosto de 2004, constituídas de 10 arrastos cada. Após a captura, os peixes foram imediatamente fixados em formol $10 \%$.

Os peixes foram identificados segundo Román (1978), Figueiredo \& Menezes (1978), Figueiredo \& Menezes (1980), Menezes \& Figueiredo (1980), Menezes \& Figueiredo (1985) e Figueiredo \& Menezes (2000), sendo classificados conforme Nelson (1994). Após a identificação, os peixes foram depositados na coleção ictiológica de referência do Laboratório de Ecologia de Peixes Tropicais da Universidade de Pernambuco.

Foi medido o comprimento padrão (CP) dos indivíduos em centímetros. Devido ao elevado número de exemplares de Ophioscion punctatissimus Meek \& Hildebrand, 1925 (família Sciaenidae) capturados, a distribuição de freqüência de comprimentos desta espécie foi analisada.

Os dados de precipitação pluviométrica mensal foram fornecidos pelo Instituto Nacional de Meteorologia (Instituto Tecnológico de Pernambuco, 2008).

A diversidade das espécies foi medida através do índice de diversidade específica de Shannon $\left(H^{\prime}\right)$, na base 10 , calculada pela fórmula $H^{\prime}=-\sum_{i=1}^{k} p i \log p i$, onde $k=$ número de espécies e $p i=$ proporção das observações encontradas na espécie. A uniformidade da distribuição das espécies foi medida pelo índice de equitabilidade de Shannon $\left(E_{H}\right)$, calculado por $E_{H^{\prime}}=H^{\prime} / H^{\prime}{ }_{\max }$. Os índices foram calculados com o auxílio do programa computacional Ecologia (Ludwig \& REYNOLDS, 1988). A densidade foi estimada por $D=\left(\sum N I i 100\right) / A$,

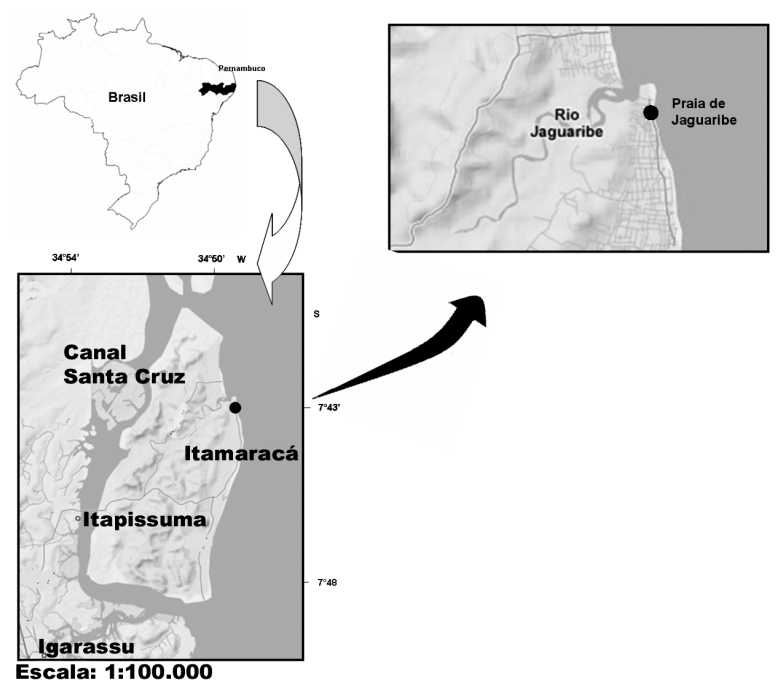

Fig. 1. Mapa da ilha de Itamaracá, Pernambuco, com a indicação do local de coleta na praia de Jaguaribe $(\bullet)$. sendo $N I$ o número de indivíduos da espécie $i$ e $A$ a área; e a biomassa por $B=\left(\sum P T i 100\right) / A$, sendo PT o peso total dos indivíduos da espécie $i$ e $A$ a área.

Os testes de Shapiro Wilk e de Bartlett foram procedidos para analisar, respectivamente, a normalidade e homocedasticidade do número de indivíduos (ZAR, 1996). E, após esta análise, foi utilizado o teste de KruskalWallis para comparar a densidade de indivíduos capturados nos períodos seco/chuvoso, ao nível de significância 0,05 (ZAR, 1996).

\section{RESULTADOS}

Foi coletado um total de 417 peixes, dentre os quais se identificou representantes de sete ordens, 15 famílias, 24 gêneros e 25 espécies. As ordens identificadas foram Perciformes $(82,25 \%)$, Clupeiformes $(16,55 \%)$, Atheriniformes $(0,24 \%)$, Beloniformes $(0,24 \%)$, Gasterosteiformes (0,24\%), Siluriformes (0,24\%) e Tetraodontiformes $(0,24 \%)$, sendo que as ordens Clupeiformes e Perciformes estiveram presentes em todas as coletas (Tab. I).

A ordem Perciformes esteve representada por sete famílias, 15 gêneros e 16 espécies. O maior número de famílias da ordem Perciformes em uma mesma coleta ocorreu no mês de abril de 2003. Dentro desta ordem, a família Sciaenidae, representada por Ophioscion punctatissimus, Larimus breviceps Cuvier, 1830, Stellifer rastrifer (Jordan, 1889), Pareques acuminatus (Bloch \& Schneider, 1801), Isopisthus parvipinnis (Cuvier, 1830) e Umbrina coroides Cuvier, 1830, foi a que se destacou, representando 49,40\% de todas as amostras com 206 indivíduos (Tab. I).

Outra família com alta representatividade, dentre os Perciformes e no total, foi Polynemidae, que ocorreu nas coletas de abril e novembro de 2003 , com $49(33,33 \%)$ e 17 (26,98\%) indivíduos, respectivamente. Esta família apresentou apenas uma espécie, Polydactylus virginicus (Linnaeus, 1758) e foi responsável por $15,83 \%$ do total das amostras (Tab. I).

A família Carangidae apresentou 42 (10,07\%) indivíduos no total e esteve representada por Trachinotus carolinus (Linnaeus, 1766), Trachinotus falcatus (Linnaeus, 1758), Carangoides bartholomaei (Cuvier, 1833) e Selene vomer (Linnaeus, 1758) (Tab. I).

A família Clupeidae (Clupeiformes) esteve representada por Chirocentrodon bleekerianus (Poey, 1867) apenas na coleta de agosto de 2004, com 34 indivíduos, representando $51,52 \%$ do total coletado neste mês e $8,15 \%$ do total de indivíduos amostrados, sendo a quarta em número de exemplares (Tab. I).

A família Engraulidae (Clupeiformes) foi composta pelas espécies Lycengraulis grossidens (Agassiz, 1829) e Anchoa tricolor (Spix \& Agassiz, 1829), sendo a quinta família em número de indivíduos, 32, representando 7,67\% do total de indivíduos coletados.

Representantes das famílias Lutjanidae (Lutjanus sp.), Scaridae (Sparisoma sp. e Scarus sp.) e Pristigasteridae (Pellona harroweri (Fowler, 1917)) foram capturados em apenas uma das coletas com, 
respectivamente, $5(1,20 \%), 5(1,20 \%)$ e $3(0,72 \%)$ indivíduos do total amostrado (Tab. I).

A freqüência relativa total das famílias Centropomidae (Centropomus undecimalis (Bloch, 1792)), Atherinopsidae (Atherinella brasiliensis (Quoy \& Gaimard, 1825)), Ariidae (Cathorops spixii (Agassiz, 1829)), Tetraodontidae (Sphoeroides testudineus (Linnaeus, 1758)), Hemiramphidae (Hemiramphus brasiliensis (Linnaeus, 1758)) e Syngnathidae (Cosmocampus elucens (Poey, 1868)) foi de 0,24\%, visto que estas somente estiveram representadas em uma coleta e com apenas um exemplar cada (Tab. I).
A espécie Ophioscion punctatissimus, da família Sciaenidae, foi a mais representativa, com 170 indivíduos. Esta contabilizou $40,77 \%$ do total de peixes coletados, $49,56 \%$ do total de Perciformes e $82,52 \%$ dos representantes da família Sciaenidae, estando presente nas quatro coletas e alcançando seu maior percentual na coleta de março de 2004, com 64,54\% (91 exemplares) (Tab. I).

A distribuição de frequiência de comprimentos padrão de $O$. punctatissimus apresentou distribuição bimodal, sugerindo a formação de pulsos de recrutamento (Fig. 2A). No ano de 2003, a média do comprimento padrão foi de $4,9 \mathrm{~cm}$ e a moda foi de $5,3 \mathrm{~cm}$, enquanto que no ano

Tab. I. Densidade, biomassa (g), número total (n), comprimento padrão médio $(\overline{\mathrm{CP}})$ e desvio padrão (DP) das espécies de peixes coletadas na praia de Jaguaribe, Itamaracá, Pernambuco, 2003/2004. Números entre parênteses indicam as freqüências relativas do número de indivíduos capturados por família.

\begin{tabular}{|c|c|c|c|c|c|c|c|c|}
\hline \multirow{3}{*}{ Ordem } & \multirow{3}{*}{ Família } & \multirow{3}{*}{ Espécie } & \multicolumn{4}{|c|}{ Densidade/Biomassa } & \multirow{3}{*}{$\mathrm{n}$} & \multirow{3}{*}{$\overline{\mathrm{CP}}(\mathrm{cm}) \pm \mathrm{DP}$} \\
\hline & & & Abr & Nov & Mar & Ago & & \\
\hline & & & 2003 & 2003 & 2004 & 2004 & & \\
\hline \multirow[t]{4}{*}{ Clupeiformes } & Engraulidae & Anchoa tricolor & $3,6 / 8,1$ & & & & 2 & $4,6 \pm 2,9$ \\
\hline & $(7,67 \%)$ & Lycengraulis grossidens & $8,9 / 115,3$ & $17,8 / 28,2$ & $16,0 / 17,2$ & $1,7 / 4,8$ & 30 & $5,1 \pm 2,8$ \\
\hline & $\begin{array}{c}\text { Pristigasteridae } \\
(0,72 \%)\end{array}$ & Pellona harroweri & & & & $5,3 / 4,6$ & 3 & $4,0 \pm 0,2$ \\
\hline & $\begin{array}{c}\text { Clupeidae } \\
(8,15 \%)\end{array}$ & Chirocentrodon bleekerianus & & & & $6,4 / 55,8$ & 34 & $4,8 \pm 0,8$ \\
\hline Siluriformes & $\begin{array}{l}\text { Ariidae } \\
(0,24 \%)\end{array}$ & Cathorops spixii & $1,8 / 148,7$ & & & & 1 & 19,3 \\
\hline Atheriniformes & $\begin{array}{c}\text { Atherinopsidae } \\
(0,24 \%)\end{array}$ & Atherinella brasiliensis & $1,8 / 0,3$ & & & & 1 & 2,4 \\
\hline Beloniformes & $\begin{array}{l}\text { Hemiramphidae } \\
\qquad(0,24 \%)\end{array}$ & Hemiramphus brasiliensis & & & $1,8 / 14,1$ & & 1 & 16,0 \\
\hline Gasterosteiformes & $\begin{array}{c}\text { Syngnathidae } \\
(0,24 \%)\end{array}$ & Cosmocampus elucens & & & $1,8 / 0,6$ & & 1 & 9,0 \\
\hline \multirow[t]{16}{*}{ Perciformes } & $\begin{array}{c}\text { Centropomidae } \\
(0,24 \%)\end{array}$ & Centropomus undecimalis & & & & $1,8 / 141,2$ & 1 & 9,4 \\
\hline & $\begin{array}{c}\text { Carangidae } \\
(10,07 \%)\end{array}$ & Carangoides bartholomaei & & $1,8 / 4,7$ & & & 1 & 4,5 \\
\hline & & Selene vomer & $1,8 / 4,2$ & $1,8 / 3,4$ & & & 2 & $3,9 \pm 0,5$ \\
\hline & & Trachinotus carolinus & $7,1 / 13,6$ & $19,6 / 14,8$ & $14,2 / 33,9$ & $5,3 / 3,4$ & 26 & $3,7 \pm 0,9$ \\
\hline & & Trachinotus falcatus & $3,6 / 7,3$ & $12,4 / 136,1$ & $7,1 / 32,4$ & & 13 & $5,5 \pm 2,0$ \\
\hline & $\begin{array}{c}\text { Lutjanidae } \\
(1,20 \%)\end{array}$ & Lutjanus sp. & $8,9 / 0,9$ & & & & 5 & $2,0 \pm 0,3$ \\
\hline & $\begin{array}{c}\text { Haemulidae } \\
(4,32 \%)\end{array}$ & Conodon nobilis & $16,0 / 73,8$ & $5,3 / 12,4$ & $10,7 / 22,5$ & & 18 & $4,7 \pm 1,3$ \\
\hline & $\begin{array}{c}\text { Polynemidae } \\
(15,83 \%)\end{array}$ & Polydactylus virginicus & $87,1 / 229,1$ & $3,2 / 49,7$ & & & 66 & $4,8 \pm 1,0$ \\
\hline & $\begin{array}{c}\text { Sciaenidae } \\
(49,40 \%)\end{array}$ & Isopisthus parvipinnis & & & & $1,8 / 8,7$ & 1 & 7,8 \\
\hline & & Larimus breviceps & $17,8 / 11,3$ & & $32,0 / 108,6$ & $5,3 / 30,1$ & 31 & $4,4 \pm 1,3$ \\
\hline & & Ophioscion punctatissimus & $94,2 / 293,7$ & $21,3 / 60,8$ & $161,8 / 399,9$ & $24,9 / 45,1$ & 170 & $4,6 \pm 1,0$ \\
\hline & & Pareques acuminatus & & & $1,8 / 1,5$ & & 1 & 3,4 \\
\hline & & Stellifer rastrifer & & & $1,8 / 2,3$ & $1,8 / 2,6$ & 2 & $4,0 \pm 0,8$ \\
\hline & & Umbrina coroides & & & $1,8 / 1,4$ & & 1 & 3,2 \\
\hline & $\begin{array}{l}\text { Scaridae } \\
(1,20 \%)\end{array}$ & Sparisoma sp. & $7,1 / 6,0$ & & & & 4 & $3,4 \pm 0,4$ \\
\hline & & Scarus sp. & $1,8 / 1,5$ & & & & 1 & 3,2 \\
\hline \multirow[t]{2}{*}{ Tetraodontiformes } & $\begin{array}{c}\text { Tetraodontidae } \\
(0,24 \%)\end{array}$ & Sphoeroides testudineus & & $1,8 / 4,2$ & & & 1 & 4,0 \\
\hline & & $\mathrm{n}$ & 147 & 63 & 141 & 66 & 417 & \\
\hline
\end{tabular}


de 2004 esses valores foram, respectivamente, $4,4 \mathrm{~cm}$ e 4,1 cm (Figs. 2B e 2C).

Os dados de precipitação pluviométrica mensal foram $116,1 \mathrm{~mm}$ em abril de 2003; 26,8 $\mathrm{mm}$ em novembro de 2003; 168,6 mm em março de 2004 e 138,9 mm em agosto de2004, caracterizando o mês de novembro como seco e os demais como chuvosos (Fig. 3).

Com relação à diversidade $\left(H^{\prime}\right)$ e à equiitabilidade $\left(E_{H}\right)$, estas foram maiores no período mais seco ( $H^{\prime}$ novembro/2003 $\left.=0,805\right)$ e menores no período mais chuvoso $\left(H^{\prime}\right.$ março/2004=0,562).

Os valores de densidade média ( \pm desvio padrão) para as coletas de abril e novembro de 2003 e março e agosto de 2004 foram, respectivamente, 18,67 $( \pm 30,95)$ ind. $/ 100 \mathrm{~m}^{2}, 9,44( \pm 8,32)$ ind. $/ 100 \mathrm{~m}^{2}, 21,97(47,34)$ ind. $/ 100 \mathrm{~m}^{2}$
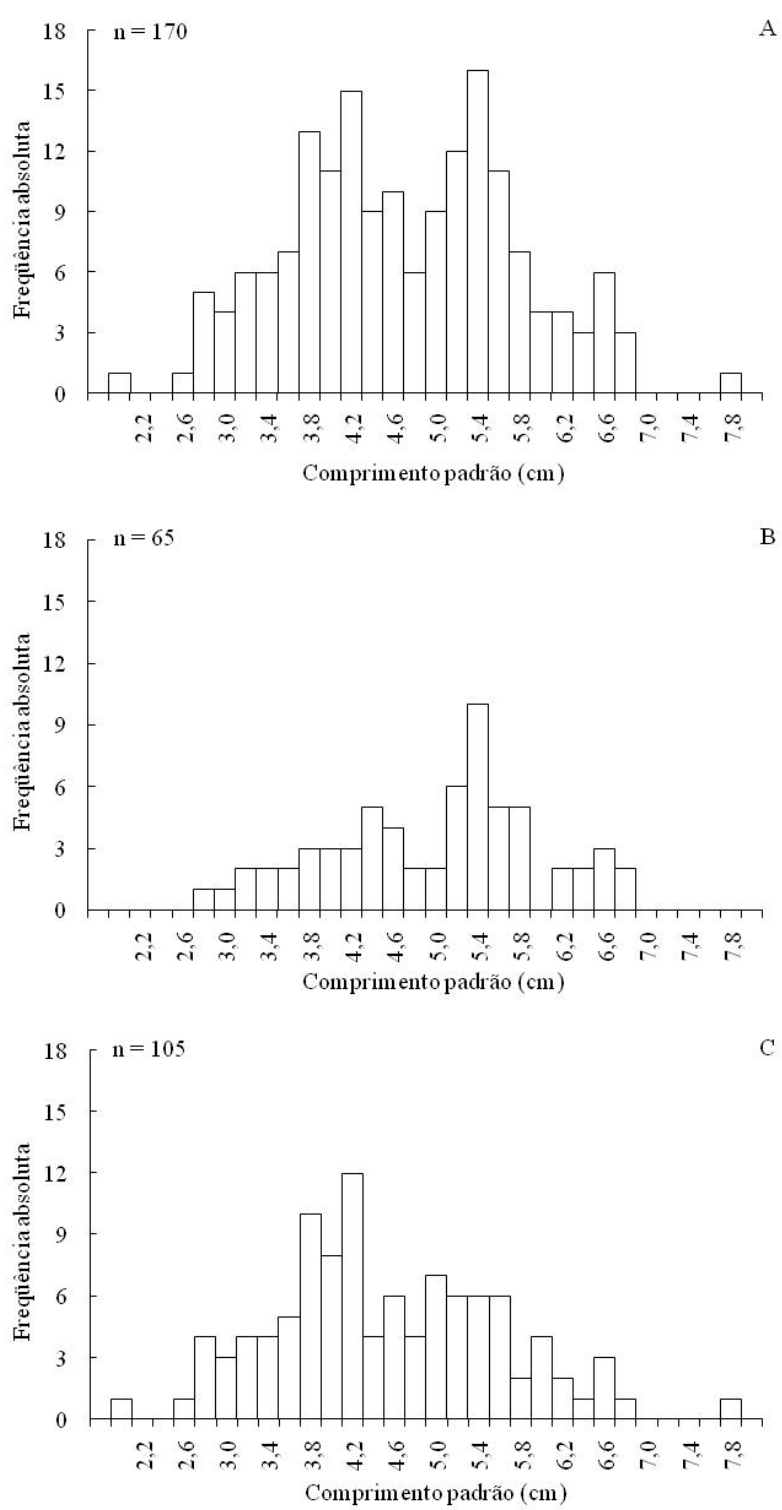

Fig. 2. Distribuição da freqüência absoluta do comprimento padrão de Ophioscion punctatissimus Meek \& Hildebrand, 1925, na praia de Jaguaribe, Itamaracá, Pernambuco, em 2003/2004. (A, total de indivíduos; B, indivíduos coletados em 2003; C, indivíduos coletados em 2004).
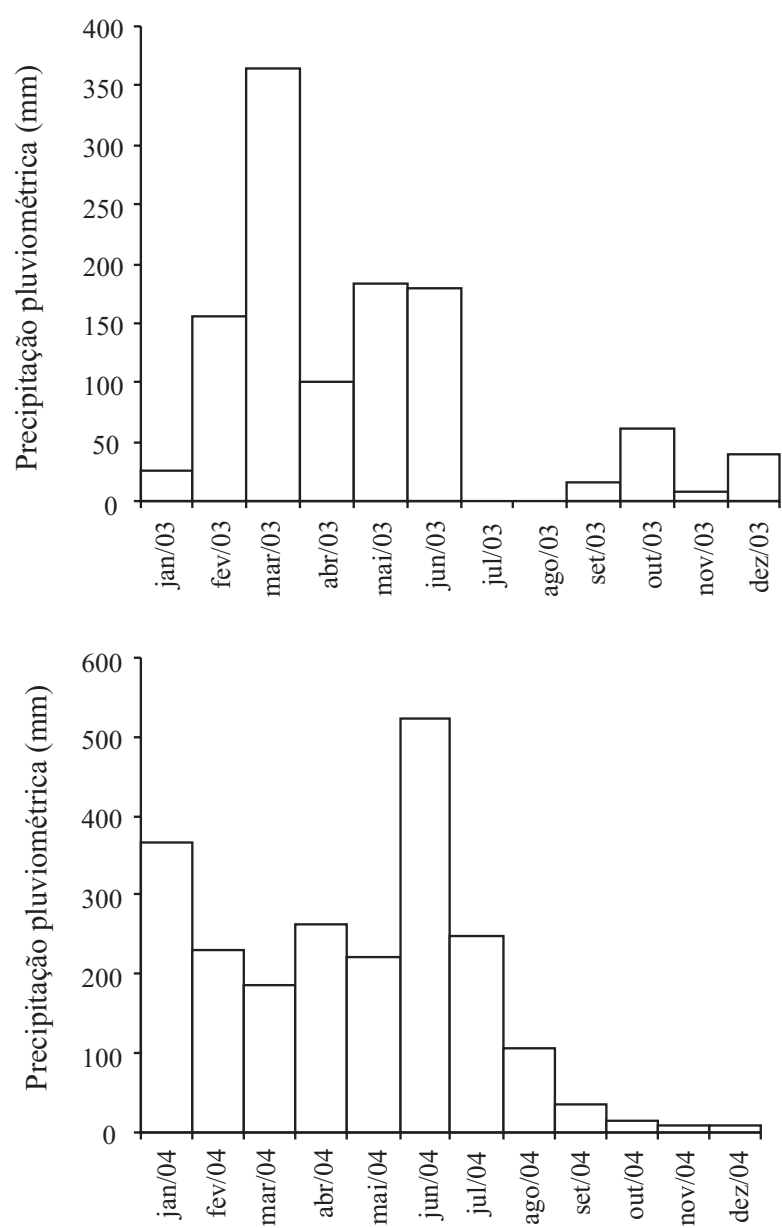

Fig. 3. Precipitação pluviométrica mensal (mm) dos anos de 2003 e 2004 em Itamaracá, Pernambuco.

e $6,04( \pm 7,33)$ ind./100 $\mathrm{m}^{2}$. A densidade por amostra não apresentou diferença significativa entre o período seco/ chuvoso $(K W=0,1827 ; P=0,6744)$. Com relação à biomassa, também não houve diferença significativa entre o período seco e o chuvoso $(K W=0,1507 ; P=0,7028)$. Os valores de biomassa média ( \pm desvio padrão) para as coletas de abril e novembro de 2003 e março e agosto de 2004 foram, respectivamente, $6,27( \pm 96,25) \mathrm{g} / 100 \mathrm{~m}^{2}, 34,92$ $( \pm 43,20) \mathrm{g} / 100 \mathrm{~m}^{2}, 56,67( \pm 117,62) \mathrm{g} / 100 \mathrm{~m}^{2} \mathrm{e} 32,92( \pm 45,24)$ $\mathrm{g} / 100 \mathrm{~m}^{2}$.

Embora não tenha ocorrido diferença significativa na abundância entre os períodos, pode se observar uma tendência de maior abundância no período chuvoso e maior diversidade no período seco.

\section{DISCUSSÃO}

A maior representatividade da ordem Perciformes corrobora as afirmações de VAsconcelos Filho et al. (2004). Dentre as famílias desta ordem, a mais representativa foi a Sciaenidae, seguida de Polynemidae e Carangidae. Vasconcelos Filho et al. (2004) também verificaram, para o canal de Santa Cruz, que as famílias Sciaenidae e Carangidae são as mais freqüentes. Sciaenidae esteve presente nas quatro coletas e as 
espécies mais freqüentes e abundantes foram Ophioscion punctatissimus e Larimus breviceps.

De acordo com Menezes \& Figueiredo (1980), Ophioscion punctatissimus vive em fundos lodosos e arenosos, em águas costeiras de pouca profundidade. A espécie não tem importância comercial e é pouco comum no litoral brasileiro (Froese \& Pauly, 2006). Para Pernambuco, apesar da sua grande abundância, também não há registro de uso comercial dessa espécie.

A espécie Larimus breviceps esteve presente em três das coletas (abril de 2003 e março e agosto de 2004) e, conforme FiguEIREDo \& MENEZES (1980), é encontrada em águas litorâneas, geralmente em profundidades inferiores a $50 \mathrm{~m}$.

A família Polynemidae predomina em águas rasas do Atlântico Oeste, em profundidades inferiores a $20 \mathrm{~m}$, sendo encontrada em águas com alta turbidez e depósitos de lama (Longhurst \& PAUly, 1987). No entanto, são importantes na região mais afastada da costa, juntamente com espécies como Larimus breviceps (Sciaenidae) e Selene vomer (Carangidae) (Longhurst \& PAUly, 1987). A família Polynemidae, representada pela espécie Polydactylus virginicus, habitante de águas rasas, esteve presente na plataforma continental da Paraíba (RAmos, 1994), bem como na praia de Jaguaribe.

As famílias Clupeidae e Engraulidae foram as mais freqüentes da ordem Clupeiformes, que foi a segunda ordem mais abundante. Estas duas famílias são as mais representativas desta ordem na região costeira de Pernambuco (VAsconcelos Filho et al., 2004).

Clupeidae, presente apenas na última coleta, foi representada exclusivamente por Chirocentrodon bleekerianus que, segundo FigueIREDo \& MENEZEs (1978), é pouco comum no litoral brasileiro. No entanto, esta espécie foi a mais freqüente na coleta de agosto de 2004, representando mais da metade dos peixes coletados, indicando que ela é um importante componente da ictiofauna de Jaguaribe sazonalmente.

A família Engraulidae, bem como a Sciaenidae e a Carangidae, esteve presente em todas as coletas, sendo representada apenas por Lycengraulis grossidens. De acordo com EKAU et al. (2001), as larvas da família Engraulidae são encontradas no canal de Santa Cruz principalmente no período de menor precipitação. Isto explica o fato da coleta de novembro ter apresentado o maior número de jovens desta família, visto que este foi o mês com menor precipitação.

Segundo Vasconcelos Filho et al. (2004), as espécies encontradas no canal de Santa Cruz podem ser classificadas ecologicamente em residentes, marinhas dependentes e marinhas visitantes. Foram consideradas espécies residentes aquelas que passam todo seu ciclo de vida no estuário. As únicas espécies residentes do canal de Santa Cruz encontradas na praia de Jaguaribe foram Atherinella brasiliensis e Sphoeroides testudineus, provavelmente pela proximidade do local das coletas com o estuário. Atherinella brasiliensis mostrou-se abundante no complexo estuarino da baía de Paranaguá (FAVAro et al., 2003) e, segundo Figueiredo \& MEnezes (2000), essa espécie é encontrada em água salobra. As espécies marinhas dependentes são aquelas que têm origem no mar e que obrigatoriamente utilizam as águas estuarinas para alimentação e reprodução. Destas, Anchoa tricolor, Centropomus undecimalis, Lycengraulis grossidens, Polydactylus virginicus e Stellifer rastrifer estiveram presentes na praia de Jaguaribe.

São consideradas espécies marinhas visitantes aquelas que têm origem no mar, porém só penetram nos estuários ocasionalmente. Dentre estas, foram encontradas em Jaguaribe Hemiramphus brasiliensis, Cosmocampus elucens, Selene vomer, Trachinotus falcatus e Conodon nobilis.

De acordo com Fonteles Filho (1989), o recrutamento consiste no processo pelo qual indivíduos produzidos por uma classe etária anual se tornam disponíveis e passíveis de identificação e quantificação, em certo estágio do ciclo vital. Um dos modos de determinar o recrutamento é acompanhando o deslocamento mensal das modas de comprimento (Fonteles Filho, 1989). Em Jaguaribe, embora as coletas não tenham sido mensais, foi possível a observação de pulsos de recrutamento para a espécie Ophioscion punctatissimus. Por meio da análise separada da distribuição da frequiência de comprimento para os anos de 2003 e 2004, observou-se que em 2003 a moda e a média do comprimento padrão foram maiores do que em 2004, sugerindo pulsos de recrutamento anual para esta espécie.

A diversidade da ictiofauna na praia de Jaguaribe apresentou variações ao longo do tempo em consequiência da precipitação pluviométrica. A coleta de novembro, mês com menor precipitação, obteve a maior diversidade e eqüitabilidade. Não houve predominância de nenhuma espécie neste mês, porém o número de peixes coletados foi o menor, com apenas 63 exemplares. A coleta com a menor diversidade foi a do mês de março de 2004, com maior precipitação, na qual só $O$. punctatissimus foi responsável por mais da metade das espécies (64,54\%). Segundo BARREIRos et al. (2004), a diversidade de peixes em poças de maré na praia Vermelha e São Roque, em Santa Catarina, também foi maior nos meses de verão.

Ainda que não tenha sido observada diferença significativa entre os períodos seco e chuvoso, a maior abundância de peixes ocorreu no período chuvoso, concordando com VENDEL et al. (2003), que encontraram variações temporais em assembléias de peixes de uma planície de maré na baía de Paranaguá.

Pôde-se observar que a ictiofauna da praia de Jaguaribe apresentou uma tendência de maior abundância no período chuvoso e maior diversidade no período seco, sugerindo que as variações pluviométricas afetam a comunidade de peixes local.

Agradecimentos. A MSc. Susmara Silva Campos e Viviane Gleice da Silva pelo suporte nas coletas de campo e a Susmara S. Campos pela revisão crítica e sugestões. Aos dois consultores anônimos pelos valiosos comentários que contribuíram para a melhoria do trabalho.

\section{REFERÊNCIAS BIBLIOGRÁFICAS}

Andrade, G. O. \& Lins, R. C. 1971. Os climas do Nordeste. In: V Asconcelos Sobrinho, J. ed. As regiões naturais do nordeste, o meio e a civilização. Recife, CONDEPE. p.95-138.

Azevedo, S. B. \& Guedes, D. S. 1980. Estudo Ecológico da Região 
de Itamaracá, Pernambuco, Brasil. X. Novas Ocorrências de Peixes. Trabalhos Oceanográficos da UFPE 15:331-341.

Barreiros, J. P.; Bertoncini, A.; Machado, L.; Hostim-Silva, M. \& SAntos, R. S. 2004. Diversity and seasonal changes in the ichthyofauna of rocky tidal pools from Praia Vermelha and São Roque, Santa Catarina. Brazilian Archives of Biology and Technology 47(2):291-299.

Cavalcanti, L. B. 1979. Estudo Ecológico da Região de Itamaracá, Pernambuco, Brasil. I. Nota Introdutória. Trabalhos Oceanográficos UFPE 14:55-64.

Cavalcanti, E. F.; Vasconcelos-Filho, A. L.; Souza, S. T. \& Guedes, D. S. 1998. Novas ocorrências de peixes para o Canal de Santa Cruz - Itamaracá, PE. Caderno Ômega UFRPE (Série Ciências Aquáticas) 4:7-16.

Couto, L. M. M. R. \& Vasconcelos Filho, A. L. 1980. Estudo Ecológico da Região de Itamaracá, Pernambuco, Brasil. VIII. Sobre a Biologia de Chaetodipterus faber (Broussonet, 1982), Pisces, Eppiphidae, no Canal de Santa Cruz. Trabalhos Oceanográficos da UFPE 15:311-321.

Ekau, W.; Westhaus-Ekau, P.; Macêdo, S. J. \& Dorrien, C. V. 2001. The larval fish of the "Canal de Santa Cruz" - estuary in northeast Brazil. Tropical Oceanography 29(2):1-12.

EskinaZi-Lece, E. \& Vasconcelos Filho, A. L. 1972. Diatomáceas no conteúdo estomacal de Mugil spp. (Pisces - Mugilidae). Trabalhos Oceanográficos da UFPE 13:107-118

Favaro, L. F.; Lopes, S. C. G. \& Spach, H. L. 2003. Reprodução do peixe-rei, Atherinella brasiliensis (Quoy \& Gaimard) (Atheriniformes, Atherinopsidae), em uma planície de maré adjacente à gamboa de Baguaçu, Baía de Paranaguá, Paraná, Brasil. Revista Brasileira de Zoologia 20(3):501-506.

Figueiredo, J. L. \& Menezes, N. A. 1978. Manual de peixes marinhos do sudeste do Brasil II. Teleostei (1). São Paulo, Museu de Zoologia USP. 110p.

1980. Manual de peixes marinhos do sudeste do Brasil III. Teleostei (2). São Paulo, Museu de Zoologia USP. 90p.

2000. Manual de peixes marinhos do sudeste do Brasil VI. Teleostei (5). São Paulo, Museu de Zoologia USP. $100 \mathrm{p}$

Fonteles Filho, A. A. 1989. Recursos pesqueiros: biologia e dinâmica populacional. Fortaleza, Impressão Oficial do Ceará. 296p.

Froese, R. \& Pauly, D. 2006. Fishbase. Disponível em: <http:// www.fishbase.org>. Acesso em: 19.07.2005.

Guedes, D. S. \& Vasconcelos Filho, A. L. 1980. Estudo Ecológico da Região de Itamaracá, Pernambuco, Brasil. IX. Informações sobre a Alimentação dos Bagres Branco e Amarelo (Pisces, Ariidae). Trabalhos Oceanográficos da UFPE 15:323-330.

ITEP. 2008. Disponível em: 〈http://www.itep.br/lamepe.ASP>. Acesso em: 09.05.2008.

Longhurst, A. R. \& PAULy, D. 1987. Ecology of tropical oceans. San Diego, Academic. 407p.
Ludwig, J. A. \& Reynolds, J. F. 1988. Statistical Ecology: a Primer on Methods and Computing. New York, John Wiley \& Sons. 337p.

Macêdo, S. J.; Flores Montes, M. J. \& Lins, I. C. 2000 Características abióticas da área. In: BARros, H. M.; EskINAZILeÇA, E.; Macêdo, S. J. \& Lima, T. eds. Gerenciamento participativo de estuários e manguezais. Recife, UFPE. p.7-25.

Macêdo, S. J.; Muniz, K. \& Montes, M. J. F. 2004. Hidrologia da região costeira e plataforma continental do estado de Pernambuco. In: Eskinazi Leça, E.; Neumann Leitão, S. \& Costa, M. F. orgs. Oceanografia: um cenário tropical. Recife, Bagaço. p.255-286.

Menezes, N. A. \& Figueiredo, J. L. 1980. Manual de Peixes Marinhos do Sudeste do Brasil IV. Teleostei (3). São Paulo, Museu de Zoologia USP. 96p.

1985. Manual de peixes marinhos do sudeste do Brasil V. Teleostei (4). São Paulo, Museu de Zoologia USP. 105p.

Nelson, J. S. 1994. Fishes of the World. New York, Wiley \& Sons. 600p.

Pereira, R. C. \& Soares-Gomes, A. 2002. Biologia Marinha. Rio de Janeiro, Interciência. 382p.

Ramos, R. T. C. 1994. Análise da composição e distribuição da fauna de peixes demersais da plataforma continental da Paraíba e estados vizinhos. Revista Nordestina de Biologia 9(1):1-30.

Román, B. 1978. Peces marinos de Venezuela claves dicotomicas de las familias. Contribución $\mathrm{N}^{\circ} 74$, Estación de Investigaciones Marinas de Margarita. Madrid, Fundación La Salle de Ciências Naturales. 107p.

Vasconcelos Filho, A. L. 1979. Estudo Ecológico da Região de Itamaracá, Pernambuco, Brasil. IV. Alimentação da Sardinha Bandeira, Opisthonema oglinum (Le Sueur, 1817), no Canal de Santa Cruz. Trabalhos Oceanográficos da UFPE 14:105-116.

Vasconcelos Filho, A. L., Guedes, D. S., Teixeira, S. F. \& Eskinaz Oliveira, A. M. 2004. Peixes marinhos costeiros e estuarinos. In: Leitão, S. N.; EskinaZi-LeÇA, E. \& Costa, M. Oceanografia: um cenário tropical. Recife, Bagaço. p.555-570.

Vasconcelos Filho, A. L.; Neumann-Leitão, S.; Eskinazi-Leç, E.; Schwamborn, R.; Eskinazi-Oliveira, A. M. \& Paranagua, M. N. 2003. Trophic interactions between fish and other compartment comunities in a tropical estuary in Brazil as indicator of environmental quality. In: Tiezzi, E.; BrebBia, C. A. \& Usó, J. L. eds. Ecosystems and Sustainable Development IV. Southampton, Wit, v.1. p.173-183

Vasconcelos Filho, A. L. \& Oliveira, A. M. E. 1999. Composição e ecologia da ictiofauna do Canal de Santa Cruz (Itamaracá - PE, Brasil). Trabalhos Oceanográficos da UFPE 27(1):101-113.

Vendel, A. L.; Lopes, S. G.; Santos, C. \& Spach, H. L. 2003. Fish assemblages in a tidal flats. Brazilian Archives of Biology and Technology 46(2):233-242.

ZaR, J. H. 1996. Biostatistical Analysis. New Jersey, PrenticeHall. $662 \mathrm{p}$

Recebido em outubro de 2005. Aceito em julho de 2008. ISSN 0073-4721

Artigo disponível em: www.scielo.br/isz 